\title{
Tribonacci and Tribonacci-Lucas Hybrinomials
}

\author{
Yasemin Taşyurdu ${ }^{1} \&$ Yunis Emre Polat ${ }^{2}$ \\ ${ }^{1}$ Department of Mathematics, Faculty of Science and Art, Erzincan Binali Yıldırım University, Erzincan, Turkey \\ ${ }^{2}$ Department of Mathematics, Graduate School of Natural and Applied Sciences, Erzincan Binali Yıldırım University, \\ Erzincan, Turkey \\ Correspondence: Yasemin Taşyurdu, Department of Mathematics, Faculty of Science and Art, Erzincan Binali Yıldırım \\ University, 24100 Erzincan, Turkey.
}

\author{
Received: May 10, 2021 Accepted: August 25, 2021 Online Published: September 29, 2021 \\ doi:10.5539/jmr.v13n5p32 URL: https://doi.org/10.5539/jmr.v13n5p32
}

\begin{abstract}
In this paper, we introduce Tribonacci and Tribonacci-Lucas hybrinomials and derive these hybrinomials by the matrices. We present Binet formulas, generating functions, exponential generating functions and summation formulas, some properties of these hybrinomials. Moreover, we obtain relationship between the Tribonacci and Tribonacci-Lucas hybrinomials.
\end{abstract}

Keywords: Tribonacci polynomial, Tribonacci-Lucas polynomial, Tribonacci hybrinomial, Tribonacci-Lucas hybrinomial

2010 Mathematics Subject Classification: 11B83, 11B37, 05A15

\section{Introduction}

A recursive sequence, also known as a recurrence sequence, is usually defined by a recurrence procedure; that is, any term of this sequence is the sum of preceding terms and generated by solving a recurrence equation. Fibonacci sequence, which is a sequence of integers, is the most famous second order sequence in all science with interesting properties. In particular, many various generalizations of this sequence have been studied in the literature. (see, for example, McCarty, 1981; Pethe, 1988; Koshy, 2001; Gupta et al, 2012a; Taşyurdu, 2016). The Tribonacci and Tribonacci-Lucas sequences, which are the well-known generalizations of Fibonacci sequences, are third order recurrence sequences. In 1963, Feinberg originally studied the Tribonacci sequence (Feinberg, 1963). For $n \geq 3$, the Tribonacci sequence $\left\{T_{n}\right\}_{n \geq 0}$ is defined by

$$
T_{n}=T_{n-1}+T_{n-2}+T_{n-3}
$$

with inital conditions $T_{0}=0, T_{1}=1, T_{2}=1$ and the Tribonacci-Lucas sequence $\left\{K_{n}\right\}_{n \geq 0}$ is defined by

$$
K_{n}=K_{n-1}+K_{n-2}+K_{n-3}
$$

with inital conditions $K_{0}=3, K_{1}=1, K_{2}=3$. The Tribonacci and Tribonacci-Lucas sequences have many interesting properties and applications in many fields of science. Several authors presented the Binet formulas, generating functions, exponential generating functions, summation formulas and matrix representation of the Tribonacci and Tribonacci-Lucas sequences (see, for example, Scott et al, 1977; Shannon, 1977; Spickerman, 1981; Bruce, 1984; Taşyurdu, 2019a; Soykan, 2020).

In (Hoggatt \& Bicknell, 1973), the Tribonacci polynomials were introduced in 1973 by Hoggatt and Bicknell. For any integer $n \geq 3$, the recurrence relation of the Tribonacci polynomials is as follows

$$
T_{n}(x)=x^{2} T_{n-1}(x)+x T_{n-2}(x)+T_{n-3}(x)
$$

where $T_{0}(x)=0, T_{1}(x)=1, T_{2}(x)=x^{2}$. Obviously, $T_{n}(1)=T_{n}$ where $T_{n}$ is the $n$th classical Tribonacci number. The recurrence relation of the Tribonacci-Lucas polynomials is defined by

$$
K_{n}(x)=x^{2} K_{n-1}(x)+x K_{n-2}(x)+K_{n-3}(x)
$$

where $K_{0}(x)=3, K_{1}(x)=x^{2}, K_{2}(x)=x^{4}+2 x$. 
Table 1. The first few Tribonacci and Tribonacci-Lucas polynomials are

\begin{tabular}{l|ll}
\hline$n$ & $T_{n}(x)$ & $K_{n}(x)$ \\
\hline 0 & 0 & 3 \\
1 & 1 & $x^{2}$ \\
2 & $x^{2}$ & $x^{4}+2 x$ \\
3 & $x^{4}+x$ & $x^{6}+3 x^{3}+3$ \\
4 & $x^{6}+2 x^{3}+1$ & $x^{8}+4 x^{5}+6 x^{2}$ \\
5 & $x^{8}+3 x^{5}+3 x^{2}$ & $x^{10}+5 x^{7}+10 x^{4}+5 x$ \\
6 & $x^{10}+4 x^{7}+6 x^{4}+2 x$ & $x^{12}+6 x^{9}+15 x^{6}+14 x^{3}+3$, \\
7 & $x^{12}+5 x^{9}+10 x^{6}+7 x^{3}+1$ & $x^{14}+7 x^{11}+21 x^{8}+28 x^{5}+14 x^{2}$ \\
8 & $x^{14}+6 x^{11}+15 x^{8}+16 x^{5}+6 x^{2}$ & $x^{16}+8 x^{13}+28 x^{10}+48 x^{7}+38 x^{4}+8 x$ \\
\hline
\end{tabular}

Using standard techniques for solving recurrence relations, the roots of the characteristic equation $\lambda^{3}-x^{2} \lambda^{2}-x \lambda-1=$ 0 of equations (1) and (2) are $\alpha_{1}(x), \alpha_{2}(x)$ and $\alpha_{3}(x)$ such that

$$
\begin{aligned}
& \alpha_{1}(x)=\frac{x^{2}}{3}+\sqrt[3]{\frac{x^{6}}{27}+\frac{x^{3}}{6}+\frac{1}{2}+\sqrt{\frac{x^{6}}{37}+\frac{7 x^{3}}{54}+\frac{1}{4}}}+\sqrt[3]{\frac{x^{6}}{27}+\frac{x^{3}}{6}+\frac{1}{2}-\sqrt{\frac{x^{6}}{37}+\frac{7 x^{3}}{54}+\frac{1}{4}}} \\
& \alpha_{2}(x)=\frac{x^{2}}{3}+\omega \sqrt[3]{\frac{x^{6}}{27}+\frac{x^{3}}{6}+\frac{1}{2}+\sqrt{\frac{x^{6}}{37}+\frac{7 x^{3}}{54}+\frac{1}{4}}}+\omega^{2} \sqrt[3]{\frac{x^{6}}{27}+\frac{x^{3}}{6}+\frac{1}{2}-\sqrt{\frac{x^{6}}{37}+\frac{7 x^{3}}{54}+\frac{1}{4}}} \\
& \alpha_{3}(x)=\frac{x^{2}}{3}+\omega^{2} \sqrt[3]{\frac{x^{6}}{27}+\frac{x^{3}}{6}+\frac{1}{2}+\sqrt{\frac{x^{6}}{37}+\frac{7 x^{3}}{54}+\frac{1}{4}}}+\omega \sqrt[3]{\frac{x^{6}}{27}+\frac{x^{3}}{6}+\frac{1}{2}-\sqrt{\frac{x^{6}}{37}+\frac{7 x^{3}}{54}+\frac{1}{4}}},
\end{aligned}
$$

with $\omega=\frac{-1+i \sqrt{3}}{2}$. Then, the Binet formulas for the Tribonacci and Tribonacci-Lucas polynomials are given by

$$
T_{n}(x)=\frac{\alpha_{1}^{n+1}(x)}{\left(\alpha_{1}(x)-\alpha_{2}(x)\right)\left(\alpha_{1}(x)-\alpha_{3}(x)\right)}+\frac{\alpha_{2}{ }^{n+1}(x)}{\left(\alpha_{2}(x)-\alpha_{1}(x)\right)\left(\alpha_{2}(x)-\alpha_{3}(x)\right)}+\frac{\alpha_{3}{ }^{n+1}(x)}{\left(\alpha_{3}(x)-\alpha_{1}(x)\right)\left(\alpha_{3}(x)-\alpha_{2}(x)\right)}
$$

and

$$
K_{n}(x)=\alpha_{1}^{n}(x)+\alpha_{2}^{n}(x)+\alpha_{3}^{n}(x)
$$

respectively. Note that we have the following identities

$$
\begin{aligned}
\alpha_{1}(x)+\alpha_{2}(x)+\alpha_{3}(x) & =x^{2} \\
\alpha_{1}(x) \alpha_{2}(x) \alpha_{3}(x) & =1 .
\end{aligned}
$$

Many authors have studied the generalized Fibonacci and Tribonacci polynomials and their properties (Gupta et al, 2012b; Gültekin \& Taşyurdu, 2013; Kose et al, 2014; Ramirez \& Sirvent 2014). For $n \geq 2$, the following relation between Tribonacci polynomials and Tribonacci-Lucas polynomials is introduced

$$
K_{n}(x)=x^{2} T_{n}(x)+2 x T_{n-1}(x)+3 T_{n-2}(x)
$$

The generating functions of the Tribonacci and Tribonacci-Lucas polynomials are given by

$$
\begin{aligned}
& G(t)=\sum_{n=0}^{\infty} T_{n}(x) t^{n}=\frac{t}{1-x^{2} t-x t^{2}-t^{3}} \\
& R(t)=\sum_{n=0}^{\infty} K_{n}(x) t^{n}=\frac{3-2 x^{2} t-x t^{2}}{1-x^{2} t-x t^{2}-t^{3}}
\end{aligned}
$$

where $T_{n}(x)$ is the $n$th Tribonacci polynomial and $K_{n}(x)$ is the $n$th Tribonacci-Lucas polynomial. For $x=1$, we obtain the generating functions of the Tribonacci and Tribonacci-Lucas numbers, respectively.

On the other hand, hybrid numbers which are a new generalization of complex, hyperbolic and dual numbers have 
applications in different areas of mathematics. Moreover, these numbers have been extensively used in science, engineering and theoretical physics. Özdemir (Özdemir, 2018) introduced a new non-commutative number system called hybrid numbers. The hybrid number system can be considered as a generalization of the complex, hyperbolic and dual number systems. The set of hybrid numbers, denoted by $\mathbb{K}$, is defined as

$$
\mathbb{K}=\left\{a+b i+c \varepsilon+d h: a, b, c, d \in \mathbb{R}, i^{2}=-1, \varepsilon^{2}=0, h^{2}=1, i h=-h i=\varepsilon+i\right\} .
$$

Let $Z_{1}=a+b i+c \varepsilon+d h$ and $Z_{2}=x+y i+z \varepsilon+t h$ be any two hybrid numbers. The equality, addition, substraction and multiplication by scalar are defined as follows

Equality: $\quad Z_{1}=Z_{2}$ only if $a=x, b=y, c=z, d=t$

Addition: $\quad Z_{1}+Z_{2}=(a+x)+(b+y) i+(c+z) \varepsilon+(d+t) h$

Substraction: $\quad Z_{1}-Z_{2}=(a-x)+(b-y) i+(c-z) \varepsilon+(d-t) h$

Multiplication by scalar $\lambda \in \mathbb{R}: \lambda Z_{1}=\lambda a+\lambda b i+\lambda c \varepsilon+\lambda d h$.

Table 2. The multiplication of hybrid units of $\mathbb{K}$

\begin{tabular}{ccccc}
\hline $\mathrm{x}$ & 1 & $\mathrm{i}$ & $\varepsilon$ & $\mathrm{h}$ \\
\hline 1 & 1 & $\mathrm{i}$ & $\varepsilon$ & $\mathrm{h}$ \\
$\mathrm{i}$ & $\mathrm{i}$ & -1 & $1-\mathrm{h}$ & $\varepsilon+\mathrm{i}$ \\
$\varepsilon$ & $\varepsilon$ & $1+\mathrm{h}$ & 0 & $-\varepsilon$ \\
$\mathrm{h}$ & $\mathrm{h}$ & $-\varepsilon-\mathrm{i}$ & $\varepsilon$ & 1 \\
\hline
\end{tabular}

The Table 2 present that $(\mathbb{K},+)$ is an Abelian group. This implies that the multiplication of hybrid numbers has the property of associativity. But it is not commutative. Addition of the hybrid numbers is both associative and commutative. Zero is the null element. The additive inverse of a hybrid number $Z$ is $-Z=-a-b i-c \varepsilon-d h$. The readers can find more detailed information about the hybrid numbers in (Özdemir, 2018).

Many researchs activities can be seen in resent years studies on special type of hybrid numbers. For example, Szynal-Liana and Wloch considered the Fibonacci hybrid numbers, the Pell and Pell-Lucas hybrid numbers and the Jacosthal and Jacosthal-Lucas.hybrid numbers and obtained some properties of these numbers, respectively. (Szynal-Liana \& Wloch, 2018; 2019a; 2019b). In (Szynal-Liana, 2018) Szynal-Liana generalized their results and defined the Horadam hybrid numbers. In (Cerda-Morales, 2018), Cerda-Morales defined the $n$th hybrid $(p, q)$-Fibonacci and $n$th hybrid $(p, q)$-Lucas numbers, respectively. Polatlı defined hybrid numbers with the Fibonacci and Lucas hybrid number coefficients (Polatll, 2020). Also, hybrid numbers were applied to third order recurrence sequences. Tasyurdu (Taşyurdu, 2019b) and Yağmur (Yağmur, 2020) introduced the Tribonacci and Tribonacci-Lucas hybrid numbers and expressed many various properties besides the known properties for sequences of these hybrid numbers. The $n$th Tribonacci hybrid number, $\mathbb{H} T_{n}$ and $n$th Tribonacci-Lucas hybrid number, $\mathbb{H} K_{n}$ are defined by

$$
\begin{aligned}
\mathbb{H} T_{n} & =T_{n}+T_{n+1} i+T_{n+2} \varepsilon+T_{n+3} h \\
\mathbb{H} K_{n} & =K_{n}+K_{n+1} i+K_{n+2} \varepsilon+K_{n+3} h
\end{aligned}
$$

where $T_{n}$ is the $n$th Tribonacci number and $K_{n}$ is the $n$th Tribonacci-Lucas number, respectively. Then new generalizations of the hybrid numbers were introduced, called hybrinomial sequences. In (Liana \& Szynal-Liana, 2019; Szynal-Liana \& Włoch, 2020a; 2020b) the authors introduced the Pell hybrinomials, the Fibonacci and Lucas hybrinomials and generalized Fibonacci-Pell hybrinomials, respectively. Also, Kızılateş defined the Horadam hybrinomials and gave some special cases of these hybrinomials (Kızılateş, 2020).

The aim of this study is to present a new generalization of the Tribonacci and Tribonacci-Lucas hybrid numbers, called as, Tribonacci and Tribonacci-Lucas hybrinomials, that is, polynomials, which are a generalization of the Tribonacci hybrid numbers and Tribonacci-Lucas hybrid numbers, respectively.

\section{The Tribonacci and Tribonacci-Lucas Hybrinomials}

In this section, we define the Tribonacci and Tribonacci-Lucas hybrinomials and give recurrence relations of these hybrinomials. Then we present the Binet formulas for the $n$th Tribonacci hybrinomial and $n$th Tribonacci-Lucas hybrinomial. We introduce the generating functions and exponential generating functions of these hybrinomials. Moreover, we obtain the summation formulas, some properties, matrix representation and relation between the Tribonacci and Tribonacci-Lucas hybrinomials.

Definition 2.1. The $n$th Tribonacci hybrinomial, $T_{n} \mathcal{H}(x)$ and $n$th Tribonacci-Lucas hybrinomial, $K_{n} \mathcal{H}(x)$ are defined by

$$
T_{n} \mathcal{H}(x)=T_{n}(x)+i T_{n+1}(x)+\varepsilon T_{n+2}(x)+h T_{n+3}(x)
$$




$$
K_{n} \mathcal{H}(x)=K_{n}(x)+i K_{n+1}(x)+\varepsilon K_{n+2}(x)+h K_{n+3}(x)
$$

where $T_{n}(x)$ is the $n$th Tribonacci polynomial, $K_{n}(x)$ is the $n$th Tribonacci-Lucas polynomial and hybrid units $i, \varepsilon, h$ satisfy the the equations $i^{2}=-1, \varepsilon^{2}=0, h^{2}=1, i h=-h i=\varepsilon+i$.

The Tribonacci and Tribonacci-Lucas hybrinomials satisfy a linear recurrence of degree three. Now present the recurrence relations of the Tribonacci hybrinomial sequence, $\left\{T_{n} \mathcal{H}(x)\right\}_{n \geq 0}$ and Tribonacci-Lucas hybrinomial sequence $\left\{K_{n} \mathcal{H}(x)\right\}_{n \geq 0}$.

Theorem 2.1. For $n \geq 3$, the following recurrence relations holds

$$
\begin{aligned}
T_{n} \mathcal{H}(x) & =x^{2} T_{n-1} \mathcal{H}(x)+x T_{n-2} \mathcal{H}(x)+T_{n-3} \mathcal{H}(x) \\
K_{n} \mathcal{H}(x) & =x^{2} K_{n-1} \mathcal{H}(x)+x K_{n-2} \mathcal{H}(x)+K_{n-3} \mathcal{H}(x)
\end{aligned}
$$

with

$$
\begin{aligned}
& T_{0} \mathcal{H}(x)=i+\varepsilon x^{2}+h\left(x^{4}+x\right), \\
& T_{1} \mathcal{H}(x)=1+i x^{2}+\varepsilon\left(x^{4}+x\right)+h\left(x^{6}+2 x^{3}+1\right), \\
& T_{2} \mathcal{H}(x)=x^{2}+i\left(x^{4}+x\right)+\varepsilon\left(x^{6}+2 x^{3}+1\right)+h\left(x^{8}+3 x^{5}+3 x^{2}\right)
\end{aligned}
$$

and

$$
\begin{aligned}
& K_{0} \mathcal{H}(x)=3+i x^{2}+\varepsilon\left(x^{4}+2 x\right)+h\left(x^{6}+3 x^{3}+3\right), \\
& K_{1} \mathcal{H}(x)=x^{2}+i\left(x^{4}+2 x\right)+\varepsilon\left(x^{6}+3 x^{3}+3\right)+h\left(x^{8}+4 x^{5}+6 x^{2}\right), \\
& K_{2} \mathcal{H}(x)=x^{4}+2 x+i\left(x^{6}+3 x^{3}+3\right)+\varepsilon\left(x^{8}+4 x^{5}+6 x^{2}\right)+h\left(x^{10}+5 x^{7}+10 x^{4}+5 x\right),
\end{aligned}
$$

respectively.

Proof. Using the equations (1) and (6), we obtain

$$
\begin{aligned}
T_{n} \mathcal{H}(x) & =T_{n}(x)+i T_{n+1}(x)+\varepsilon T_{n+2}(x)+h T_{n+3}(x) \\
& =x^{2} T_{n-1}(x)+x T_{n-2}(x)+T_{n-3}(x)+i\left(x^{2} T_{n}(x)+x T_{n-1}(x)+T_{n-2}(x)\right) \\
& +\varepsilon\left(x^{2} T_{n+1}(x)+x T_{n}(x)+T_{n-1}(x)\right)+h\left(x^{2} T_{n+2}(x)+x T_{n+1}(x)+T_{n}(x)\right) \\
& =x^{2}\left(T_{n-1}(x)+i T_{n}(x)+\varepsilon T_{n+1}(x)+h T_{n+2}(x)\right) \\
& +x\left(T_{n-2}(x)+i T_{n-1}(x)+\varepsilon T_{n}(x)+h T_{n+1}(x)\right) \\
& +T_{n-3}(x)+i T_{n-2}(x)+\varepsilon T_{n-1}(x)+h T_{n}(x) \\
& =x^{2} T_{n-1} \mathcal{H}(x)+x T_{n-2} \mathcal{H}(x)+T_{n-3} \mathcal{H}(x) .
\end{aligned}
$$

Similarly, we can obtain $K_{n} \mathcal{H}(x)=x^{2} K_{n-1} \mathcal{H}(x)+x K_{n-2} \mathcal{H}(x)+K_{n-3} \mathcal{H}(x)$ using the equations (2) and (7).

Using Teorem 2.1, it can be easily shown that the Tribonacci and Tribonacci-Lucas hybrinomial sequences can be extended to negative subscripts by the recurrence relations as follows

$$
\begin{aligned}
& T_{-n} \mathcal{H}(x)=-x T_{-(n-1)} \mathcal{H}(x)-x^{2} T_{-(n-2)} \mathcal{H}(x)+T_{-(n-3)} \mathcal{H}(x) \\
& K_{-n} \mathcal{H}(x)=-x K_{-(n-1)} \mathcal{H}(x)-x^{2} K_{-(n-2)} \mathcal{H}(x)+K_{-(n-3)} \mathcal{H}(x)
\end{aligned}
$$

for $n \geq 1$, respectively.

\subsection{The Binet Formulas and Generating Functions of the Tribonacci and Tribonacci-Lucas Hybrinomials}

The Tribonacci and Tribonacci-Lucas hybrinomials can be obtained by using the Definition 2.1 and Theorem 2.1. The Binet formula known as the general formula can be used instead of both definition and theorem. Now, we produce the Binet formulas for the Tribonacci and Tribonacci-Lucas hybrinomials.

Theorem 2.2. Let $n \geq 0$ be an integer and $\alpha_{1}(x), \alpha_{2}(x)$ and $\alpha_{3}(x)$ are the roots of the characteristic equation $\lambda^{3}-x^{2} \lambda^{2}-x \lambda-1=0$. Then the Binet formulas for the Tribonacci and Tribonacci-Lucas hybrinomials are given by

$$
\begin{aligned}
T_{n} \mathcal{H}(x) & =\frac{\hat{\alpha}_{1}(x) \alpha_{1}{ }^{n+1}(x)}{\left(\alpha_{1}(x)-\alpha_{2}(x)\right)\left(\alpha_{1}(x)-\alpha_{3}(x)\right)}+\frac{\hat{\alpha}_{2}(x) \alpha_{2}{ }^{n+1}(x)}{\left(\alpha_{2}(x)-\alpha_{1}(x)\right)\left(\alpha_{2}(x)-\alpha_{3}(x)\right)}+\frac{\hat{\alpha}_{3}(x) \alpha_{3}{ }^{n+1}(x)}{\left(\alpha_{3}(x)-\alpha_{1}(x)\right)\left(\alpha_{3}(x)-\alpha_{2}(x)\right)} \\
K_{n} \mathcal{H}(x) & =\widehat{\alpha}_{1}(x) \alpha_{1}{ }^{n}(x)+\widehat{\alpha}_{2}(x) \alpha_{2}{ }^{n}(x)+\widehat{\alpha}_{3}(x) \alpha_{3}{ }^{n}(x)
\end{aligned}
$$

respectively, where $\hat{\alpha}_{1}(x)=1+i \alpha_{1}(x)+\varepsilon \alpha_{1}{ }^{2}(x)+h \alpha_{1}{ }^{3}(x), \hat{\alpha}_{2}(x)=1+i \alpha_{2}(x)+\varepsilon \alpha_{2}{ }^{2}(x)+h \alpha_{2}{ }^{3}(x), \hat{\alpha}_{3}(x)=$ $1+i \alpha_{3}(x)+\varepsilon \alpha_{3}{ }^{2}(x)+h \alpha_{3}{ }^{3}(x)$. Moreover, 


$$
\begin{aligned}
& \alpha_{1}(x)=\frac{x^{2}}{3}+\sqrt[3]{\frac{x^{6}}{27}+\frac{x^{3}}{6}+\frac{1}{2}+\sqrt{\frac{x^{6}}{37}+\frac{7 x^{3}}{54}+\frac{1}{4}}}+\sqrt[3]{\frac{x^{6}}{27}+\frac{x^{3}}{6}+\frac{1}{2}-\sqrt{\frac{x^{6}}{37}+\frac{7 x^{3}}{54}+\frac{1}{4}}} \\
& \alpha_{2}(x)=\frac{x^{2}}{3}+\omega \sqrt[3]{\frac{x^{6}}{27}+\frac{x^{3}}{6}+\frac{1}{2}+\sqrt{\frac{x^{6}}{37}+\frac{7 x^{3}}{54}+\frac{1}{4}}+\omega^{2} \sqrt[3]{\frac{x^{6}}{27}+\frac{x^{3}}{6}+\frac{1}{2}-\sqrt{\frac{x^{6}}{37}+\frac{7 x^{3}}{54}+\frac{1}{4}}}} \\
& \alpha_{3}(x)=\frac{x^{2}}{3}+\omega^{2} \sqrt[3]{\frac{x^{6}}{27}+\frac{x^{3}}{6}+\frac{1}{2}+\sqrt{\frac{x^{6}}{37}+\frac{7 x^{3}}{54}+\frac{1}{4}}}+\omega \sqrt[3]{\frac{x^{6}}{27}+\frac{x^{3}}{6}+\frac{1}{2}-\sqrt{\frac{x^{6}}{37}+\frac{7 x^{3}}{54}+\frac{1}{4}}}
\end{aligned}
$$

with $\omega=\frac{-1+i \sqrt{3}}{2}$.

Proof. By considering the Binet formula for the $n$th Tribonacci polynomial given in equation (3) and equation (6), we have

$$
\begin{aligned}
T_{n} \mathcal{H}(x) & =T_{n}(x)+i T_{n+1}(x)+\varepsilon T_{n+2}(x)+h T_{n+3}(x) \\
& =\frac{\alpha_{1}{ }^{n+1}(x)}{\left(\alpha_{1}(x)-\alpha_{2}(x)\right)\left(\alpha_{1}(x)-\alpha_{3}(x)\right)}+\frac{\alpha_{2}{ }^{n+1}(x)}{\left(\alpha_{2}(x)-\alpha_{1}(x)\right)\left(\alpha_{2}(x)-\alpha_{3}(x)\right)}+\frac{\alpha_{3}{ }^{n+1}(x)}{\left(\alpha_{3}(x)-\alpha_{1}(x)\right)\left(\alpha_{3}(x)-\alpha_{2}(x)\right)} \\
& +i\left(\frac{\alpha_{1}{ }^{n+2}(x)}{\left(\alpha_{1}(x)-\alpha_{2}(x)\right)\left(\alpha_{1}(x)-\alpha_{3}(x)\right)}+\frac{\alpha_{2}{ }^{n+2}(x)}{\left(\alpha_{2}(x)-\alpha_{1}(x)\right)\left(\alpha_{2}(x)-\alpha_{3}(x)\right)}+\frac{\alpha_{3}{ }^{n+2}(x)}{\left(\alpha_{3}(x)-\alpha_{1}(x)\right)\left(\alpha_{3}(x)-\alpha_{2}(x)\right)}\right) \\
& +\varepsilon\left(\frac{\alpha_{1}{ }^{n+3}(x)}{\left(\alpha_{1}(x)-\alpha_{2}(x)\right)\left(\alpha_{1}(x)-\alpha_{3}(x)\right)}+\frac{\alpha_{2}{ }^{n+3}(x)}{\left(\alpha_{2}(x)-\alpha_{1}(x)\right)\left(\alpha_{2}(x)-\alpha_{3}(x)\right)}+\frac{\alpha_{3}{ }^{n+3}(x)}{\left(\alpha_{3}(x)-\alpha_{1}(x)\right)\left(\alpha_{3}(x)-\alpha_{2}(x)\right)}\right) \\
& +h\left(\frac{\alpha_{1}{ }^{n+4}(x)}{\left(\alpha_{1}(x)-\alpha_{2}(x)\right)\left(\alpha_{1}(x)-\alpha_{3}(x)\right)}+\frac{\alpha_{2}{ }^{n+4}(x)}{\left(\alpha_{2}(x)-\alpha_{1}(x)\right)\left(\alpha_{2}(x)-\alpha_{3}(x)\right)}+\frac{\alpha_{3}{ }^{n+4}(x)}{\left(\alpha_{3}(x)-\alpha_{1}(x)\right)\left(\alpha_{3}(x)-\alpha_{2}(x)\right)}\right) \\
& =\frac{\left(1+i \alpha_{1}(x)+\varepsilon \alpha_{1}{ }^{2}(x)+h \alpha_{1}{ }^{3}(x)\right) \alpha_{1}{ }^{n+1}(x)}{\left(\alpha_{1}(x)-\alpha_{2}(x)\right)\left(\alpha_{1}(x)-\alpha_{3}(x)\right)}+\frac{\left(1+i \alpha_{2}(x)+\varepsilon \alpha_{2}{ }^{2}(x)+h \alpha_{2}{ }^{3}(x)\right) \alpha_{2}{ }^{n+1}(x)}{\left(\alpha_{2}(x)-\alpha_{1}(x)\right)\left(\alpha_{2}(x)-\alpha_{3}(x)\right)} \\
& +\frac{\left(1+i \alpha_{3}(x)+\varepsilon \alpha_{3}{ }^{2}(x)+h \alpha_{3}{ }^{3}(x)\right) \alpha_{3}{ }^{n+1}(x)}{\left(\alpha_{3}(x)-\alpha_{1}(x)\right)\left(\alpha_{3}(x)-\alpha_{2}(x)\right)} \\
& =\frac{\widehat{\alpha}_{1}(x) \alpha_{1}{ }^{n+1}(x)}{\left(\alpha_{1}(x)-\alpha_{2}(x)\right)\left(\alpha_{1}(x)-\alpha_{3}(x)\right)}+\frac{\widehat{\alpha}_{2}(x) \alpha_{2}{ }^{n+1}(x)}{\left(\alpha_{2}(x)-\alpha_{1}(x)\right)\left(\alpha_{2}(x)-\alpha_{3}(x)\right)}+\frac{\widehat{\alpha}_{3}(x) \alpha_{3}{ }^{n+1}(x)}{\left(\alpha_{3}(x)-\alpha_{1}(x)\right)\left(\alpha_{3}(x)-\alpha_{2}(x)\right)}
\end{aligned}
$$

and using the Binet formula for the $n$th Tribonacci-Lucas polynomial given in equation (4) and equation (7), we have

$$
\begin{aligned}
K_{n} \mathcal{H}(x) & =K_{n}(x)+i K_{n+1}(x)+\varepsilon K_{n+2}(x)+h K_{n+3}(x) \\
& =\alpha_{1}{ }^{n}(x)+\alpha_{2}{ }^{n}(x)+\alpha_{3}{ }^{n}(x)+i\left(\alpha_{1}{ }^{n+1}(x)+\alpha_{2}{ }^{n+1}(x)+\alpha_{3}{ }^{n+1}(x)\right) \\
& +\varepsilon\left(\alpha_{1}{ }^{n+2}(x)+\alpha_{2}{ }^{n+2}(x)+\alpha_{3}{ }^{n+2}(x)\right)+h\left(\alpha_{1}{ }^{n+3}(x)+\alpha_{2}{ }^{n+3}(x)+\alpha_{3}{ }^{n+3}(x)\right) \\
& =\left(1+i \alpha_{1}(x)+\varepsilon \alpha_{1}{ }^{2}(x)+h \alpha_{1}{ }^{3}(x)\right) \alpha_{1}{ }^{n}(x) \\
& +\left(1+i \alpha_{2}(x)+\varepsilon \alpha_{2}{ }^{2}(x)+h \alpha_{2}{ }^{3}(x)\right) \alpha_{2}{ }^{n}(x) \\
& +\left(1+i \alpha_{3}(x)+\varepsilon \alpha_{3}{ }^{2}(x)+h \alpha_{3}{ }^{3}(x)\right) \alpha_{3}{ }^{n}(x) \\
& =\hat{\alpha}_{1}(x) \alpha_{1}{ }^{n}(x)+\hat{\alpha}_{2}(x) \alpha_{2}{ }^{n}(x)+\hat{\alpha}_{3}(x) \alpha_{3}{ }^{n}(x)
\end{aligned}
$$

where

$$
\begin{aligned}
& \widehat{\alpha}_{1}(x)=1+i \alpha_{1}(x)+\varepsilon \alpha_{1}{ }^{2}(x)+h \alpha_{1}{ }^{3}(x), \\
& \widehat{\alpha}_{2}(x)=1+i \alpha_{2}(x)+\varepsilon \alpha_{2}{ }^{2}(x)+h \alpha_{2}{ }^{3}(x), \\
& \widehat{\alpha}_{3}(x)=1+i \alpha_{3}(x)+\varepsilon \alpha_{3}{ }^{2}(x)+h \alpha_{3}{ }^{3}(x) .
\end{aligned}
$$


which ends the proof.

As it is known, the generating functions are one of the most powerful techniques for solving linear recurrence relations. Now, we give the generating functions for the Tribonacci and Tribonacci-Lucas hybrinomials. We know that the power series of the ordinary generating function for $\left\{a_{0}, a_{1}, a_{3} \ldots\right\}$ are follows

$$
g(t)=a_{0}+a_{1} t+a_{2} t^{2}+\cdots+a_{n} t^{n}+\cdots=\sum_{n=0}^{\infty} a_{n} t^{n} .
$$

Theorem 2.3. The generating functions for the Tribonacci and Tribonacci-Lucas hybrinomial sequences are

$$
\begin{aligned}
& g(t)=\sum_{n=0}^{\infty} T_{n} \mathcal{H}(x) t^{n}=\frac{T_{0} \mathcal{H}(x)+\left(T_{1} \mathcal{H}(x)-x^{2} T_{0} \mathcal{H}(x)\right) t+T_{-1} \mathcal{H}(x) t^{2}}{1-x^{2} t-x t^{2}-t^{3}} \\
& r(t)=\sum_{n=0}^{\infty} K_{n} \mathcal{H}(x) t^{n}=\frac{K_{0} \mathcal{H}(x)+\left(K_{1} \mathcal{H}(x)-x^{2} K_{0} \mathcal{H}(x)\right) t+K_{-1} \mathcal{H}(x) t^{2}}{1-x^{2} t-x t^{2}-t^{3}}
\end{aligned}
$$

respectively.

Proof. Let $g(t)=\sum_{n=0}^{\infty} T_{n} \mathcal{H}(x) t^{n}$ be the generating function for the Tribonacci hybrinomial sequence. Then

$$
\begin{aligned}
g(t) & =\sum_{n=0}^{\infty} T_{n} \mathcal{H}(x) t^{n} \\
& =T_{0} \mathcal{H}(x)+T_{1} \mathcal{H}(x) t+\sum_{n=2}^{\infty} T_{n} \mathcal{H}(x) t^{n} \\
& =T_{0} \mathcal{H}(x)+T_{1} \mathcal{H}(x) t+\sum_{n=2}^{\infty}\left(x^{2} T_{n-1} \mathcal{H}(x)+x T_{n-2} \mathcal{H}(x)+T_{n-3} \mathcal{H}(x)\right) t^{n} \\
& =T_{0} \mathcal{H}(x)+T_{1} \mathcal{H}(x) t+x^{2} t \sum_{n=0}^{\infty} T_{n} \mathcal{H}(x) t^{n}-x^{2} T_{0} \mathcal{H}(x) t \\
& +x t^{2} \sum_{n=0}^{\infty} T_{n}(x) \mathcal{H} t^{n}+t^{3} \sum_{n=0}^{\infty} T_{n} \mathcal{H}(x) t^{n}+T_{-1} \mathcal{H}(x) t^{2} \\
& =T_{0} \mathcal{H}(x)+T_{1} \mathcal{H}(x) t+x^{2} t g(t)-x^{2} T_{0} \mathcal{H}(x) t+x t^{2} g(t)+t^{3} g(t)+T_{-1} \mathcal{H}(x) t^{2}
\end{aligned}
$$

and we obtain that

$$
\left(1-x^{2} t-x t^{2}-t^{3}\right) g(t)=T_{0} \mathcal{H}(x)+T_{1} \mathcal{H}(x) t-x^{2} T_{0} \mathcal{H}(x) t+T_{-1} \mathcal{H}(x) t^{2} .
$$

So the generating function for the Tribonacci hybrinomial sequence is

$$
g(t)=\frac{T_{0} \mathcal{H}(x)+\left(T_{1} \mathcal{H}(x)-x^{2} T_{0} \mathcal{H}(x)\right) t+T_{-1} \mathcal{H}(x) t^{2}}{1-x^{2} t-x t^{2}-t^{3}} .
$$

and similarly, it can easily prove the generating function for the Tribonacci-Lucas hybrinomial sequence as follows

$$
r(t)=\frac{K_{0} \mathcal{H}(x)+\left(K_{1} \mathcal{H}(x)-x^{2} K_{0} \mathcal{H}(x)\right) t+K_{-1} \mathcal{H}(x) t^{2}}{1-x^{2} t-x t^{2}-t^{3}} .
$$

Theorem 2.4. For $m \geq 2$, the generating functions for the the Tribonacci hybrinomial sequence $\left\{T_{n+m} \mathcal{H}(x)\right\}_{n \geq 0}$ and Tribonacci-Lucas hybrinomial sequence $\left\{K_{n+m} \mathcal{H}(x)\right\}_{n \geq 0}$ are

$$
\begin{aligned}
& \sum_{n=0}^{\infty} T_{n+m} \mathcal{H}(x) t^{n}=\frac{T_{m} \mathcal{H}(x)+\left(x T_{m-1} \mathcal{H}(x)-T_{m-2} \mathcal{H}(x)\right) t+T_{m-1} \mathcal{H}(x) t^{2}}{1-x^{2} t-x t^{2}-t^{3}} \\
& \sum_{n=0}^{\infty} K_{n+m} \mathcal{H}(x) t^{n}=\frac{K_{m} \mathcal{H}(x)+\left(x K_{m-1} \mathcal{H}(x)-K_{m-2} \mathcal{H}(x)\right) t+K_{m-1} \mathcal{H}(x) t^{2}}{1-x^{2} t-x t^{2}-t^{3}}
\end{aligned}
$$

respectively. 
Proof. By using the Binet formula for the $n$th Tribonacci hybrinomial given in equation (10), we get

$$
\begin{aligned}
& \sum_{n=0}^{\infty} T_{n+m} \mathcal{H}(x) t^{n}=\sum_{n=0}^{\infty}\left(\frac{\hat{\alpha}_{1}(x) \alpha_{1}{ }^{n+m+1}(x)}{\left(\alpha_{1}(x)-\alpha_{2}(x)\right)\left(\alpha_{1}(x)-\alpha_{3}(x)\right)}+\frac{\hat{\alpha}_{2}(x) \alpha_{2}{ }^{n+m+1}(x)}{\left(\alpha_{2}(x)-\alpha_{1}(x)\right)\left(\alpha_{2}(x)-\alpha_{3}(x)\right)}+\frac{\hat{\alpha}_{3}(x) \alpha_{3}{ }^{n+m+1}(x)}{\left(\alpha_{3}(x)-\alpha_{1}(x)\right)\left(\alpha_{3}(x)-\alpha_{2}(x)\right)}\right) t^{n} \\
& =\frac{\widehat{\alpha}_{1}(x) \alpha_{1}^{m+1}(x)}{\left(\alpha_{1}(x)-\alpha_{2}(x)\right)\left(\alpha_{1}(x)-\alpha_{3}(x)\right)} \sum_{n=0}^{\infty}\left(\alpha_{1}(x) t\right)^{n} \\
& +\frac{\widehat{\alpha}_{2}(x) \alpha_{2}{ }^{m+1}(x)}{\left(\alpha_{2}(x)-\alpha_{1}(x)\right)\left(\alpha_{2}(x)-\alpha_{3}(x)\right)} \sum_{n=0}^{\infty}\left(\alpha_{2}(x) t\right)^{n} \\
& +\frac{\widehat{\alpha}_{3}(x) \alpha_{3}{ }^{m+1}(x)}{\left(\alpha_{3}(x)-\alpha_{1}(x)\right)\left(\alpha_{3}(x)-\alpha_{2}(x)\right)} \sum_{n=0}^{\infty}\left(\alpha_{3}(x) t\right)^{n} \\
& =\frac{\widehat{\alpha}_{1}(x) \alpha_{1}^{m+1}(x)}{\left(\alpha_{1}(x)-\alpha_{2}(x)\right)\left(\alpha_{1}(x)-\alpha_{3}(x)\right)}\left(\frac{1}{1-\alpha_{1}(x) t}\right) \\
& +\frac{\widehat{\alpha}_{2}(x) \alpha_{2}{ }^{m+1}(x)}{\left(\alpha_{2}(x)-\alpha_{1}(x)\right)\left(\alpha_{2}(x)-\alpha_{3}(x)\right)}\left(\frac{1}{1-\alpha_{2}(x) t}\right) \\
& +\frac{\widehat{\alpha}_{3}(x) \alpha_{3}{ }^{m+1}(x)}{\left(\alpha_{3}(x)-\alpha_{1}(x)\right)\left(\alpha_{3}(x)-\alpha_{2}(x)\right)}\left(\frac{1}{1-\alpha_{3}(x) t}\right) \\
& =\frac{1}{\left(1-\alpha_{1}(x) t\right)\left(1-\alpha_{2}(x) t\right)\left(1-\alpha_{3}(x) t\right)}\left[\left(\frac{\widehat{\alpha}_{1}(x)\left(\alpha_{1}{ }^{m+1}(x)-\alpha_{1}{ }^{m+1}(x) \alpha_{3}(x)-\alpha_{1}{ }^{m+1}(x) \alpha_{2}(x)+\alpha_{1}{ }^{m}(x)\right)}{\left(\alpha_{1}(x)-\alpha_{2}(x)\right)\left(\alpha_{1}(x)-\alpha_{3}(x)\right)}\right)\right. \\
& +\left(\frac{\widehat{\alpha}_{2}(x)\left(\alpha_{2}^{m+1}(x)-\alpha_{2}^{m+1}(x) \alpha_{3}(x)-\alpha_{2}{ }^{m+1}(x) \alpha_{1}(x)+\alpha_{2}{ }^{m}(x)\right)}{\left(\alpha_{2}(x)-\alpha_{1}(x)\right)\left(\alpha_{2}(x)-\alpha_{3}(x)\right)}\right) \\
& \left.+\left(\frac{\hat{\alpha}_{3}(x)\left(\alpha_{3}{ }^{m+1}(x)-\alpha_{3}{ }^{m+1}(x) \alpha_{2}(x)-\alpha_{3}{ }^{m+1}(x) \alpha_{1}(x)+\alpha_{3}{ }^{m}(x)\right)}{\left(\alpha_{3}(x)-\alpha_{1}(x)\right)\left(\alpha_{3}(x)-\alpha_{2}(x)\right)}\right)\right]
\end{aligned}
$$

If we rearrange the last equation using the equations $\alpha_{1}(x)+\alpha_{2}(x)+\alpha_{3}(x)=x^{2}, \alpha_{1}(x) \alpha_{2}(x) \alpha_{3}(x)=1$, then we get

$$
\begin{aligned}
\sum_{n=0}^{\infty} T_{n+m} \mathcal{H}(x) t^{n} & =\frac{1}{1-x^{2} t-x t^{2}-t^{3}}\left[\left(\frac{\widehat{\alpha}_{1}(x) \alpha_{1}{ }^{m}(x)}{\left(\alpha_{1}(x)-\alpha_{2}(x)\right)\left(\alpha_{1}(x)-\alpha_{3}(x)\right)}+\frac{\widehat{\alpha}_{2}(x) \alpha_{2}{ }^{m}(x)}{\left(\alpha_{2}(x)-\alpha_{1}(x)\right)\left(\alpha_{2}(x)-\alpha_{3}(x)\right)}+\frac{\widehat{\alpha}_{3}(x) \alpha_{3}{ }^{m}(x)}{\left(\alpha_{3}(x)-\alpha_{1}(x)\right)\left(\alpha_{3}(x)-\alpha_{2}(x)\right)}\right)\right. \\
& +\left(x\left(\frac{\widehat{\alpha}_{1}(x) \alpha_{1}{ }^{m-1}(x)}{\left(\alpha_{1}(x)-\alpha_{2}(x)\right)\left(\alpha_{1}(x)-\alpha_{3}(x)\right)}+\frac{\widehat{\alpha}_{2}(x) \alpha_{2}{ }^{m-1}(x)}{\left(\alpha_{2}(x)-\alpha_{1}(x)\right)\left(\alpha_{2}(x)-\alpha_{3}(x)\right)}+\frac{\widehat{\alpha}_{3}(x) \alpha_{3}{ }^{m-1}(x)}{\left(\alpha_{3}(x)-\alpha_{1}(x)\right)\left(\alpha_{3}(x)-\alpha_{2}(x)\right)}\right)\right. \\
& \left.+\left(\frac{\widehat{\alpha}_{1}(x) \alpha_{1}{ }^{m-2}(x)}{\left(\alpha_{1}(x)-\alpha_{2}(x)\right)\left(\alpha_{1}(x)-\alpha_{3}(x)\right)}+\frac{\widehat{\alpha}_{2}(x) \alpha_{2}{ }^{m-2}(x)}{\left(\alpha_{2}(x)-\alpha_{1}(x)\right)\left(\alpha_{2}(x)-\alpha_{3}(x)\right)}+\frac{\widehat{\alpha}_{3}(x) \alpha_{3}{ }^{m-2}(x)}{\left(\alpha_{3}(x)-\alpha_{1}(x)\right)\left(\alpha_{3}(x)-\alpha_{2}(x)\right)}\right)\right) t \\
& \left.+\left(\frac{\widehat{\alpha}_{1}(x) \alpha_{1}{ }^{m-1}(x)}{\left(\alpha_{1}(x)-\alpha_{2}(x)\right)\left(\alpha_{1}(x)-\alpha_{3}(x)\right)}+\frac{\widehat{\alpha}_{2}(x) \alpha_{2}{ }^{m-1}(x)}{\left(\alpha_{2}(x)-\alpha_{1}(x)\right)\left(\alpha_{2}(x)-\alpha_{3}(x)\right)}+\frac{\widehat{\alpha}_{3}(x) \alpha_{3}{ }^{m-1}(x)}{\left(\alpha_{3}(x)-\alpha_{1}(x)\right)\left(\alpha_{3}(x)-\alpha_{2}(x)\right)}\right) t^{2}\right] \\
& =\frac{T_{m} \mathcal{H}(x)+\left(x T_{m-1} \mathcal{H}(x)-T_{m-2} \mathcal{H}(x)\right) t+T_{m-1} \mathcal{H}(x) t^{2}}{1-x^{2} t-x t^{2}-t^{3}}
\end{aligned}
$$

and similarly, using the Binet formula for the $n$th Tribonacci-Lucas hybrinomial given in equation (11) we have

$$
\sum_{n=0}^{\infty} K_{n+m} \mathcal{H}(x) t^{n}=\frac{K_{m} \mathcal{H}(x)+\left(x K_{m-1} \mathcal{H}(x)-K_{m-2} \mathcal{H}(x)\right) t+K_{m-1} \mathcal{H}(x) t^{2}}{1-x^{2} t-x t^{2}-t^{3}}
$$

So proof is completed.

Theorem 2.5. For $n \geq 0$, the exponential generating functions for the Tribonacci and Tribonacci-Lucas hybrinomial 
sequences are

$$
\begin{gathered}
\sum_{n=0}^{\infty} T_{n} \mathcal{H}(x) \frac{t^{n}}{n !}=\frac{\hat{\alpha}_{1}(x) \alpha_{1}(x) e^{\alpha_{1}(x) t}}{\left(\alpha_{1}(x)-\alpha_{2}(x)\right)\left(\alpha_{1}(x)-\alpha_{3}(x)\right)}+\frac{\hat{\alpha}_{2}(x) \alpha_{2}(x) e^{\alpha_{2}(x) t}}{\left(\alpha_{2}(x)-\alpha_{1}(x)\right)\left(\alpha_{2}(x)-\alpha_{3}(x)\right)}+\frac{\hat{\alpha}_{3}(x) \alpha_{3}(x) e^{\alpha_{3}(x) t}}{\left(\alpha_{3}(x)-\alpha_{1}(x)\right)\left(\alpha_{3}(x)-\alpha_{2}(x)\right)} \\
\sum_{n=0}^{\infty} K_{n}(x) \mathcal{H} \frac{t^{n}}{n !}=\widehat{\alpha}_{1}(x) e^{\alpha_{1}(x) t}+\widehat{\alpha}_{2}(x) e^{\alpha_{2}(x) t}+\widehat{\alpha}_{3}(x) e^{\alpha_{3}(x) t}
\end{gathered}
$$

respectively.

Proof. By using the Binet formulas for the Tribonacci and Tribonacci-Lucas hybrinomials given in equations (10) and (11), we get

$$
\begin{aligned}
\sum_{n=0}^{\infty} T_{n} \mathcal{H}(x) \frac{t^{n}}{n !} & \left.=\sum_{n=0}^{\infty}\left(\frac{\hat{\alpha}_{1}(x) \alpha_{1}{ }^{n+1}(x)}{\left(\alpha_{1}(x)-\alpha_{2}(x)\right)\left(\alpha_{1}(x)-\alpha_{3}(x)\right)}+\frac{\hat{\alpha}_{2}(x) \alpha_{2}{ }^{n+1}(x)}{\left(\alpha_{2}(x)-\alpha_{1}(x)\right)\left(\alpha_{2}(x)-\alpha_{3}(x)\right)}+\frac{\hat{\alpha}_{3}(x) \alpha_{3}{ }^{n+1}(x)}{\left(\alpha_{3}(x)-\alpha_{1}(x)\right)\left(\alpha_{3}(x)-\alpha_{2}(x)\right)}\right) \frac{t^{n}}{n !}\right) \sum_{n=0}^{\infty} \frac{\left(\alpha_{1}(x) t\right)^{n}}{n !}+\left(\frac{\widehat{\alpha}_{2}(x) \alpha_{2}(x)}{\left(\alpha_{2}(x)-\alpha_{1}(x)\right)\left(\alpha_{2}(x)-\alpha_{3}(x)\right)}\right) \sum_{n=0}^{\infty} \frac{\left(\alpha_{2}(x) t\right)^{n}}{n !} \\
& =\left(\frac{\widehat{\alpha}_{1}(x) \alpha_{1}(x)}{\left(\alpha_{1}(x)-\alpha_{2}(x)\right)\left(\alpha_{1}(x)-\alpha_{3}(x)\right)}\right. \\
& +\left(\frac{\widehat{\alpha}_{3}(x) \alpha_{3}(x)}{\left(\alpha_{3}(x)-\alpha_{1}(x)\right)\left(\alpha_{3}(x)-\alpha_{2}(x)\right)}\right) \sum_{n=0}^{\infty} \frac{\left(\alpha_{3}(x) t\right)^{n}}{n !} \\
& =\frac{\widehat{\alpha}_{1}(x) \alpha_{1}(x) e^{\alpha_{1}(x) t}}{\left(\alpha_{1}(x)-\alpha_{2}(x)\right)\left(\alpha_{1}(x)-\alpha_{3}(x)\right)}+\frac{\widehat{\alpha}_{2}(x) \alpha_{2}(x) e^{\alpha_{2}(x) t}}{\left(\alpha_{2}(x)-\alpha_{1}(x)\right)\left(\alpha_{2}(x)-\alpha_{3}(x)\right)}+\frac{\widehat{\alpha}_{3}(x) \alpha_{3}(x) e^{\alpha_{3}(x) t}}{\left(\alpha_{3}(x)-\alpha_{1}(x)\right)\left(\alpha_{3}(x)-\alpha_{2}(x)\right)}
\end{aligned}
$$

and

$$
\begin{aligned}
\sum_{n=0}^{\infty} K_{n} \mathcal{H}(x) \frac{t^{n}}{n !} & =\sum_{n=0}^{\infty}\left(\widehat{\alpha}_{1}(x) \alpha_{1}^{n}(x)+\widehat{\alpha}_{2}(x) \alpha_{2}{ }^{n}(x)+\widehat{\alpha}_{3}(x) \alpha_{3}{ }^{n}(x)\right) \frac{t^{n}}{n !} \\
& =\hat{\alpha}_{1}(x) \sum_{n=0}^{\infty} \frac{\left(\alpha_{1}(x) t\right)^{n}}{n !}+\hat{\alpha}_{2}(x) \sum_{n=0}^{\infty} \frac{\left(\alpha_{2}(x) t\right)^{n}}{n !}+\hat{\alpha}_{3}(x) \sum_{n=0}^{\infty} \frac{\left(\alpha_{3}(x) t\right)^{n}}{n !} \\
& =\hat{\alpha}_{1}(x) e^{\alpha_{1}(x) t}+\hat{\alpha}_{2}(x) e^{\alpha_{2}(x) t}+\hat{\alpha}_{3}(x) e^{\alpha_{3}(x) t}
\end{aligned}
$$

So proof is completed.

\subsection{Some Properties of the Tribonacci and Tribonacci-Lucas Hybrinomials}

In this section, we give the summation formulas, some properties and relation between the Tribonacci and Tribonacci-Lucas hybrinomials. Also, we derive the matrix representation of the Tribonacci hybrinomials.

Theorem 2.6. The summation formulas for the Tribonacci and Tribonacci-Lucas hybrinomials are as follows

$$
\begin{aligned}
\sum_{i=0}^{n} T_{i} \mathcal{H}(x) & =\frac{T_{n+2} \mathcal{H}(x)+\left(1-x^{2}\right) T_{n+1} \mathcal{H}(x)+T_{n} \mathcal{H}(x)+x^{2} T_{0} \mathcal{H}(x)-T_{1} \mathcal{H}(x)-T_{0} \mathcal{H}(x)-T_{-1} \mathcal{H}(x)}{x+x^{2}} \\
\sum_{i=0}^{n} K_{i} \mathcal{H}(x) & =\frac{K_{n+2} \mathcal{H}(x)+\left(1-x^{2}\right) K_{n+1} \mathcal{H}(x)+K_{n} \mathcal{H}(x)+x^{2} K_{0} \mathcal{H}(x)-K_{1} \mathcal{H}(x)-K_{0} \mathcal{H}(x)-K_{-1} \mathcal{H}(x)}{x+x^{2}}
\end{aligned}
$$

respectively, for $x \in \mathbb{R} \backslash\{0,-1\}$.

Proof. Using the equation (8), we can get the following relations:

$$
\begin{gathered}
x T_{0} \mathcal{H}(x)=T_{2} \mathcal{H}(x)-x^{2} T_{1} \mathcal{H}(x)-T_{-1} \mathcal{H}(x) \\
x T_{1} \mathcal{H}(x)=T_{3} \mathcal{H}(x)-x^{2} T_{2} \mathcal{H}(x)-T_{0} \mathcal{H}(x) \\
x T_{2} \mathcal{H}(x)=T_{4} \mathcal{H}(x)-x^{2} T_{3} \mathcal{H}(x)-T_{1} \mathcal{H}(x) \\
\vdots \\
x T_{n-2} \mathcal{H}(x)=T_{n} \mathcal{H}(x)-x^{2} T_{n-1} \mathcal{H}(x)-T_{n-3} \mathcal{H}(x)
\end{gathered}
$$




$$
\begin{aligned}
x T_{n-1} \mathcal{H}(x) & =T_{n+1} \mathcal{H}(x)-x^{2} T_{n} \mathcal{H}(x)-T_{n-2} \mathcal{H}(x) \\
x T_{n} \mathcal{H}(x) & =T_{n+2} \mathcal{H}(x)-x^{2} T_{n+1} \mathcal{H}(x)-T_{n-1} \mathcal{H}(x) .
\end{aligned}
$$

If we add the equations by side by, we have

$$
\begin{aligned}
x T_{0} \mathcal{H}(x)+x T_{1} \mathcal{H}(x)+\cdots+x T_{n} \mathcal{H}(x)= & T_{n} \mathcal{H}(x)+T_{n+1} \mathcal{H}(x)+T_{n+2} \mathcal{H}(x)-x^{2}\left(\sum_{i=0}^{n+1} T_{i} \mathcal{H}(x)-T_{0} \mathcal{H}(x)\right) \\
& -T_{-1} \mathcal{H}(x)-T_{0} \mathcal{H}(x)-T_{1} \mathcal{H}(x) \\
\left(x+x^{2}\right) \sum_{i=0}^{n} T_{i} \mathcal{H}(x)= & T_{n} \mathcal{H}(x)+T_{n+1} \mathcal{H}(x)+T_{n+2} \mathcal{H}(x)-x^{2} T_{n+1} \mathcal{H}(x)+x^{2} T_{0} \mathcal{H}(x) \\
& -T_{-1} \mathcal{H}(x)-T_{0} \mathcal{H}(x)-T_{1} \mathcal{H}(x)
\end{aligned}
$$

and we obtain that

$$
\sum_{i=0}^{n} T_{i} \mathcal{H}(x)=\frac{T_{n+2} \mathcal{H}(x)+\left(1-x^{2}\right) T_{n+1} \mathcal{H}(x)+T_{n} \mathcal{H}(x)+x^{2} T_{0} \mathcal{H}(x)-T_{1} \mathcal{H}(x)-T_{0} \mathcal{H}(x)-T_{-1} \mathcal{H}(x)}{x+x^{2}}
$$

and similarly, using the equation (9), we have

$$
\sum_{i=0}^{n} K_{i} \mathcal{H}(x)=\frac{K_{n+2} \mathcal{H}(x)+\left(1-x^{2}\right) K_{n+1} \mathcal{H}(x)+K_{n} \mathcal{H}(x)+x^{2} K_{0} \mathcal{H}(x)-K_{1} \mathcal{H}(x)-K_{0} \mathcal{H}(x)-K_{-1} \mathcal{H}(x)}{x+x^{2}} .
$$

So proof is completed.

Theorem 2.7. For $n \geq 0$, we have the following equalities for the Tribonacci and Tribonacci-Lucas hybrinomials

$$
\begin{aligned}
& T_{3 n} \mathcal{H}(x)=\sum_{i=0}^{n} \sum_{j=0}^{i}\left(\begin{array}{c}
n \\
i
\end{array}\right)\left(\begin{array}{l}
i \\
j
\end{array}\right) x^{i+j} T_{i+j} \mathcal{H}(x) \\
& K_{3 n} \mathcal{H}(x)=\sum_{i=0}^{n} \sum_{j=0}^{i}\left(\begin{array}{l}
n \\
i
\end{array}\right)\left(\begin{array}{l}
i \\
j
\end{array}\right) x^{i+j} K_{i+j} \mathcal{H}(x)
\end{aligned}
$$

Proof. By using the Binet formula for the $n$th Tribonacci hybrinomial given in equation (10), we get

$$
\begin{aligned}
\sum_{i=0}^{n} \sum_{j=0}^{i}\left(\begin{array}{l}
n \\
i
\end{array}\right)\left(\begin{array}{l}
i \\
j
\end{array}\right) x^{i+j} T_{i+j} \mathcal{H}(x) & =\sum_{i=0}^{n} \sum_{j=0}^{i}\left(\begin{array}{l}
n \\
i
\end{array}\right)\left(\begin{array}{l}
i \\
j
\end{array}\right) x^{i+j}\left(\frac{\hat{\alpha}_{1}(x) \alpha_{1}{ }^{i+j+1}(x)}{\left(\alpha_{1}(x)-\alpha_{2}(x)\right)\left(\alpha_{1}(x)-\alpha_{3}(x)\right)}\right) \\
& +\sum_{i=0}^{n} \sum_{j=0}^{i}\left(\begin{array}{l}
n \\
i
\end{array}\right)\left(\begin{array}{l}
i \\
j
\end{array}\right) x^{i+j}\left(\frac{\widehat{\alpha}_{2}(x) \alpha_{2}{ }^{i+j+1}(x)}{\left(\alpha_{2}(x)-\alpha_{1}(x)\right)\left(\alpha_{2}(x)-\alpha_{3}(x)\right)}\right) \\
& +\sum_{i=0}^{n} \sum_{j=0}^{i}\left(\begin{array}{l}
n \\
i
\end{array}\right)\left(\begin{array}{l}
i \\
j
\end{array}\right) x^{i+j}\left(\frac{\widehat{\alpha}_{3}(x) \alpha_{3}{ }^{i+j+1}(x)}{\left(\alpha_{3}(x)-\alpha_{1}(x)\right)\left(\alpha_{3}(x)-\alpha_{2}(x)\right)}\right) \\
& =\left(\frac{\widehat{\alpha}_{1}(x) \alpha_{1}(x)}{\left(\alpha_{1}(x)-\alpha_{2}(x)\right)\left(\alpha_{1}(x)-\alpha_{3}(x)\right)}\right) \sum_{i=0}^{n}\left(\begin{array}{c}
n \\
i
\end{array}\right)\left(x \alpha_{1}(x)+x^{2} \alpha_{1}{ }^{2}(x)\right)^{i} \\
& +\left(\frac{\widehat{\alpha}_{2}(x) \alpha_{2}(x)}{\left(\alpha_{2}(x)-\alpha_{1}(x)\right)\left(\alpha_{2}(x)-\alpha_{3}(x)\right)}\right) \sum_{i=0}^{n}\left(\begin{array}{c}
n \\
i
\end{array}\right)\left(x \alpha_{2}(x)+x^{2} \alpha_{2}{ }^{2}(x)\right)^{i}
\end{aligned}
$$




$$
\begin{aligned}
& +\left(\frac{\widehat{\alpha}_{3} \alpha_{3}(x)}{\left(\alpha_{3}(x)-\alpha_{1}(x)\right)\left(\alpha_{3}(x)-\alpha_{2}(x)\right)}\right) \sum_{i=0}^{n}\left(\begin{array}{c}
n \\
i
\end{array}\right)\left(x \alpha_{3}(x)+x^{2}{\alpha_{3}}^{2}(x)\right)^{i} \\
& =\frac{\widehat{\alpha}_{1}(x) \alpha_{1}{ }^{3 n+1}(x)}{\left(\alpha_{1}(x)-\alpha_{2}(x)\right)\left(\alpha_{1}(x)-\alpha_{3}(x)\right)}+\frac{\widehat{\alpha}_{2}(x) \alpha_{2}{ }^{3 n+1}(x)}{\left(\alpha_{2}(x)-\alpha_{1}(x)\right)\left(\alpha_{2}(x)-\alpha_{3}(x)\right)}+\frac{\widehat{\alpha}_{3}(x) \alpha_{3}{ }^{3 n+1}(x)}{\left(\alpha_{3}(x)-\alpha_{1}(x)\right)\left(\alpha_{3}(x)-\alpha_{2}(x)\right)} \\
& =T_{3 n} \mathcal{H}(x)
\end{aligned}
$$

and using the Binet formula for the $n$th Tribonacci-Lucas hybrinomial given in equation (11), we get

$$
\begin{aligned}
\sum_{i=0}^{n} \sum_{j=0}^{i}\left(\begin{array}{l}
n \\
i
\end{array}\right)\left(\begin{array}{l}
i \\
j
\end{array}\right) x^{i+j} K_{i+j} \mathcal{H}(x)= & \sum_{i=0}^{n} \sum_{j=0}^{i}\left(\begin{array}{l}
n \\
i
\end{array}\right)\left(\begin{array}{l}
i \\
j
\end{array}\right) x^{i+j}\left(\hat{\alpha}_{1}(x) \alpha_{1}{ }^{i+j}(x)\right) \\
& +\sum_{i=0}^{n} \sum_{j=0}^{i}\left(\begin{array}{l}
n \\
i
\end{array}\right)\left(\begin{array}{l}
i \\
j
\end{array}\right) x^{i+j}\left(\hat{\alpha}_{2}(x) \alpha_{2}{ }^{i+j}(x)\right) \\
& +\sum_{i=0}^{n} \sum_{j=0}^{i}\left(\begin{array}{l}
n \\
i
\end{array}\right)\left(\begin{array}{l}
i \\
j
\end{array}\right) x^{i+j}\left(\hat{\alpha}_{3}(x) \alpha_{3}{ }^{i+j}(x)\right) \\
& =\hat{\alpha}_{1}(x) \sum_{i=0}^{n}\left(\begin{array}{c}
n \\
i
\end{array}\right)\left(x \alpha_{1}(x)+x^{2} \alpha_{1}{ }^{2}(x)\right)^{i} \\
& +\hat{\alpha}_{2}(x) \sum_{i=0}^{n}\left(\begin{array}{c}
n \\
i
\end{array}\right)\left(x \alpha_{2}(x)+x^{2} \alpha_{2}{ }^{2}(x)\right)^{i} \\
& +\hat{\alpha}_{3}(x) \sum_{i=0}^{n}\left(\begin{array}{c}
n \\
i
\end{array}\right)\left(x \alpha_{3}(x)+x^{2} \alpha_{3}{ }^{2}(x)\right)^{i} \\
& =\hat{\alpha}_{1}(x) \alpha_{1}{ }^{3 n}(x)+\hat{\alpha}_{2}(x) \alpha_{2}{ }^{3 n}(x)+\hat{\alpha}_{3}(x) \alpha_{3}{ }^{3 n}(x) \\
& =K_{3 n} \mathcal{H}(x) .
\end{aligned}
$$

So proof is completed.

Theorem 2.8. The relation between the $n$th Tribonacci hybrinomial, $T_{n} \mathcal{H}(x)$ and $n$th Tribonacci-Lucas hybrinomial, $K_{n} \mathcal{H}(x)$ is

$$
K_{n} \mathcal{H}(x)=x^{2} T_{n} \mathcal{H}(x)+2 x T_{n-1} \mathcal{H}(x)+3 T_{n-2} \mathcal{H}(x)
$$

where $n \geq 2$

Proof: By using the equations (5) and (7), we have

$$
\begin{aligned}
K_{n} \mathcal{H}(x) & =K_{n}(x)+i K_{n+1}(x)+\varepsilon K_{n+2}(x)+h K_{n+3}(x) \\
& =x^{2} T_{n}(x)+2 x T_{n-1}(x)+3 T_{n-2}(x)+i\left(x^{2} T_{n+1}(x)+2 x T_{n}(x)+3 T_{n-1}(x)\right) \\
& +\varepsilon\left(x^{2} T_{n+2}(x)+2 x T_{n+1}(x)+3 T_{n}(x)\right)+h\left(x^{2} T_{n+3}(x)+2 x T_{n+2}(x)+3 T_{n+1}(x)\right) \\
& =x^{2}\left(T_{n}(x)+i T_{n+1}(x)+\varepsilon T_{n+2}(x)+h T_{n+3}(x)\right) \\
& +2 x\left(T_{n-1}(x)+i T_{n}(x)+\varepsilon T_{n+1}(x)+h T_{n+2}(x)\right. \\
& +3\left(T_{n-2}(x)+i T_{n-1}(x)+\varepsilon T_{n}(x)+h T_{n+1}(x)\right) \\
& =x^{2} T_{n} \mathcal{H}(x)+2 x T_{n-1} \mathcal{H}(x)+3 T_{n-2} \mathcal{H}(x)
\end{aligned}
$$

which completes the proof.

Theorem 2.9. The Tribonacci hybrinomials are generated by matrix 


$$
A(x)=\left(\begin{array}{ccc}
x^{2} & x & 1 \\
1 & 0 & 0 \\
0 & 1 & 0
\end{array}\right)
$$

then

$$
Q_{T \mathcal{H}(x)} \cdot A^{n}(x)=\left(\begin{array}{lll}
T_{n+4} \mathcal{H}(x) & x T_{n+3} \mathcal{H}(x)+T_{n+2} \mathcal{H}(x) & T_{n+3} \mathcal{H}(x) \\
T_{n+3} \mathcal{H}(x) & x T_{n+2} \mathcal{H}(x)+T_{n+1} \mathcal{H}(x) & T_{n+2} \mathcal{H}(x) \\
T_{n+2} \mathcal{H}(x) & x T_{n+1} \mathcal{H}(x)+T_{n} \mathcal{H}(x) & T_{n+1} \mathcal{H}(x)
\end{array}\right)
$$

where

$$
Q_{T \mathcal{H}(x)}=\left(\begin{array}{lll}
T_{4} \mathcal{H}(x) & x T_{3} \mathcal{H}(x)+T_{2} \mathcal{H}(x) & T_{3} \mathcal{H}(x) \\
T_{3} \mathcal{H}(x) & x T_{2} \mathcal{H}(x)+T_{1} \mathcal{H}(x) & T_{2} \mathcal{H}(x) \\
T_{2} \mathcal{H}(x) & x T_{1} \mathcal{H}(x)+T_{0} \mathcal{H}(x) & T_{1} \mathcal{H}(x)
\end{array}\right)
$$

and $T_{n} \mathcal{H}(x)$ is the $n$th Tribonacci hybrinomial.

Proof: By using induction on $n$, if $n=0$, then the result is obvious. Now, assume that, equation (12) is true for all positive integers $k$, that is

$$
Q_{T \mathcal{H}(x)} \cdot A^{k}(x)=\left(\begin{array}{lll}
T_{k+4} \mathcal{H}(x) & x T_{k+3} \mathcal{H}(x)+T_{k+2} \mathcal{H}(x) & T_{k+3} \mathcal{H}(x) \\
T_{k+3} \mathcal{H}(x) & x T_{k+2} \mathcal{H}(x)+T_{k+1} \mathcal{H}(x) & T_{k+2} \mathcal{H}(x) \\
T_{k+2} \mathcal{H}(x) & x T_{k+1} \mathcal{H}(x)+T_{k} \mathcal{H}(x) & T_{k+1} \mathcal{H}(x)
\end{array}\right)
$$

where $T_{k+3} \mathcal{H}(x)=x^{2} T_{k+2} \mathcal{H}(x)+x T_{k+1} \mathcal{H}(x)+T_{k} \mathcal{H}(x)$ from equation (8), for $k \geq 0$. Then, we need to show that above equality holds for $n=k+1$. Then by induction hypothesis we obtain

$$
\begin{aligned}
Q_{T \mathcal{H}(x)} \cdot A^{k+1}(x)= & \left(Q_{T \mathcal{H}(x)} \cdot A^{k}(x)\right) \cdot A(x) \\
= & \left(\begin{array}{lll}
T_{k+4} \mathcal{H}(x) & x T_{k+3} \mathcal{H}(x)+T_{k+2} \mathcal{H}(x) & T_{k+3} \mathcal{H}(x) \\
T_{k+3} \mathcal{H}(x) & x T_{k+2} \mathcal{H}(x)+T_{k+1} \mathcal{H}(x) & T_{k+2} \mathcal{H}(x) \\
T_{k+2} \mathcal{H}(x) & x T_{k+1} \mathcal{H}(x)+T_{k} \mathcal{H}(x) & T_{k+1} \mathcal{H}(x)
\end{array}\right)\left(\begin{array}{ccc}
x^{2} & x & 1 \\
1 & 0 & 0 \\
0 & 1 & 0
\end{array}\right) \\
= & \left(\begin{array}{lll}
T_{k+5} \mathcal{H}(x) & x T_{k+4} \mathcal{H}(x)+T_{k+3} \mathcal{H}(x) & T_{k+4} \mathcal{H}(x) \\
T_{k+4} \mathcal{H}(x) & x T_{k+3} \mathcal{H}(x)+T_{k+2} \mathcal{H}(x) & T_{k+3} \mathcal{H}(x) \\
T_{k+3} \mathcal{H}(x) & x T_{k+2} \mathcal{H}(x)+T_{k+1} \mathcal{H}(x) & T_{k+2} \mathcal{H}(x)
\end{array}\right)
\end{aligned}
$$

Hence, the equation (12) holds for all $n \geq 0$.

\section{Discussion}

In this paper, the Tribonacci and Tribonacci-Lucas hybrinomials, which are a generalization of the Tribonacci hybrid numbers and Tribonacci-Lucas hybrid numbers are defined and sequences of these hybrinomials are investigated, respectively. And the recurrence relations and very important properties such as the Binet formulas, generating functions, exponential generating functions and summation formulas for these sequences are obtained. Also, some properties and relation between the Tribonacci and Tribonacci-Lucas hybrinomials are given.

\section{Acknowledgments}

Authors most grateful to the referees for their useful suggestions. The authors declare that they have no competing interests.

\section{References}

Bruce, I. (1984). A modified Tribonacci sequence. Fibonacci Quarterly, 22(3), 244-246.

Cerda-Morales, G. (2018). Investigation of Generalized Hybrid Fibonacci Numbers and Their Properties. arXiv preprint, arXiv: 1806.02231v1 (2018).

Feinberg, M. (1963). Fibonacci-Tribonacci. Fibonacci Quarterly, 1, 71-74.

Gupta, V. K., Panwar, Y. K., \& Sikhwal, O. (2012a). Generalized Fibonacci Sequences. Theoretical Mathematics \& Applications, 2(2), 115-124. 
Gupta, V. K., Panwar, Y. K., \& Sikhwal, O. (2012b). Generalized Fibonacci-Like Polynomial and its Determinantal Identities. Int. J. Contemp. Math. Sciences, 7(29), 1415-1420.

Gültekin, İ., \& Taşyurdu, Y. (2013). On Period of the Sequence of Fibonacci Polynomials Modulo m. Discrete Dynamics in Nature and Society, 2013, 3p. https://doi.org/10.1155/2013/731482

Hoggatt, Jr. V. E., \& Bicknell, M. (1973). Generalized Fibonacci Polynomials. Fibonacci Quarterly, 11, 457-465.

Kizılateş, C. (2020). A Note on Horadam Hybrinomials, preprints. https://doi.org/10.20944/preprints202001.0116.v1

Koshy, T. (2001). Fibonacci and Lucas Numbers with Applications, New York, Wiley-Interscience. https://doi.org/10.1002/9781118033067

Kose, H., Yilmaz, N., \& Taskara, N. (2014). On Properties of Tribonacci-Lucas Polynomials, arXiv: 1409.3710v1 [math.NT] 12 Sep 2014.

Liana, M., Szynal-Liana, A., \& Włoch, I. (2019). On Pell Hybrinomials, Miskolc Math. Notes, 20(2), 1051-1062. https://doi.org/10.18514/MMN.2019.2971

McCarty, C. P. (1981). A Formula for Tribonacci Numbers. The Fibonacci Quarterly, 19, 391-393.

Özdemir, M. (2018). Introduction to Hybrid Numbers. Advances in Applied Clifford Algebras, 28(11). https://doi.org/10.1007/s00006-018-0833-3

Pethe, S. (1988). Some Identities for Tribonacci Sequences. The Fibonacci Quarterly, 26, 144-151.

Polatl1, E. (2020). Hybrid Numbers with Fibonacci and Lucas Hybrid Number Coefficients, preprint, 2020.

Ramirez, J. S. \& Sirvent, V. F. (2014). Incomplete Tribonacci Numbers and Polynomials. Journal of Integer Sequences, $17,1-13$.

Scott, A., Delaney, T., \& Hoggatt, Jr. V. E. (1977). The Tribonacci Sequence. Fibonacci Quarterly, 15(3), 193- 200.

Shannon, A. (1977). Tribonacci Numbers and Pascal's Pyramid. Fibonacci Quarterly, 15(3), 268- 275.

Spickerman, W. (1981). Binet's Formula for the Tribonacci Sequence. Fibonacci Quarterly, 20, 118-120.

Soykan, Y. (2020). Summing Formulas for Generalized Tribonacci Numbers. Universal Journal of Mathematics and Applications, 3(1) 1-11. https://doi.org/10.32323/ujma.637876

Szynal-Liana, A. (2018). The Horadam Hybrid Numbers. Discuss. Math. Gen. Algebra Appl., 38(1), 91-98. Szynal-Liana A., \& Wloch I., (2018). On Pell and Pell.Lucas Hybrid Numbers, Commentat. Math., 58, 11.17, (2018a). https://doi.org/10.7151/dmgaa.1287

Szynal-Liana, A., \& Wloch I. (2019a). On Jacosthal and Jacosthal.Lucas Hybrid Numbers, Ann. Math. Sil., 33, $276-283$. https://doi.org/10.2478/amsil-2018-0009

Szynal-Liana, A., \& Wloch, I., (2019b). The Fibonacci Hybrid Numbers, Utilitas Math., 110, 3.10.

Szynal-Liana, A., \& Wloch I., (2020a). Introduction to Fibonacci and Lucas Hybrinomials, Complex Var. Elliptic Equ., 65(10), 1736-1747. https://doi.org/10.1080/17476933.2019.1681416

Szynal-Liana A., \& Wloch, I. (2020b). Generalized Fibonacci-Pell Hybrinomials, Online J. Anal. Comb., 15, 1-12.

Taşyurdu, Y., Çobanoğlu, N., \& Dilmen, Z. (2016). On The a New Family of $k$-Fibonacci Numbers. Erzincan University Journal of Science and Technology, 9(1), 95-101. https://doi.org/10.18185/eufbed.01209

Taşyurdu, Y. (2019a). On the Sums of Tribonacci and Tribonacci-Lucas Numbers, Applied Mathematical Sciences, 13(24), 1201-1208. https://doi.org/10.12988/ams.2019.910144

Taşyurdu, Y. (2019b). Tribonacci and Tribonacci-Lucas Hybrid Numbers. Int. J. Contemp. Math. Sciences, 14(4), 245-254. https://doi.org/10.12988/ijcms.2019.91124

Yağmur, T. (2020). A Note On Generalized Hybrid Tribonacci Numbers. Discussiones Mathematicae General Algebra and Applications, 40, 187-199. https://doi.org/10.7151/dmgaa.1343

\section{Copyrights}

Copyright for this article is retained by the author(s), with first publication rights granted to the journal.

This is an open-access article distributed under the terms and conditions of the Creative Commons Attribution license (http://creativecommons.org/licenses/by/4.0/). 